\title{
Ética médica e rastreamento: em quais evidências deveríamos nos apoiar?
}

\author{
Medical ethics and screening: on what evidence should we support ourselves? \\ Ética médica y tamizaje: ¿sobre qué evidências deberíamos nos apoyar?
}

Armando Henrique Norman. MSc em Antropologia Médica pela Durham University. editor.rbmfc@sbmfc.org.br (Autor correspondente)

Se o rastreamento fosse uma droga, ela já teria sido retirada do mercado. Assim, qual será o primeiro pais a parar com as mamografias para rastreamento de câncer de mama?

(Peter C. Gøtzsche) ${ }^{1}$

Nesta edição a RBMFC discute o tema da ética médica, espinha dorsal que orienta tanto as demandas por serviços ou tecnologias em saúde, como a prática dos médicos de família e comunidade. Como estímulo à essa reflexão, a seçáo Debate discute a "mamografia-obrigatória preventiva" no Uruguai, enquanto que na seção Ensaios, Jamoulle e Gomez discorrem sobre o conceito de prevenção quaternária, ação que tem como objetivo oferecer alternativas eticamente aceitáveis aos usuários, de modo a prevenir o excesso de intervenções médicas. ${ }^{2}$ Portanto, apesar das consideráveis transformaçóes tecnológicas e sociais que afetam diretamente a saúde das pessoas, a ética em medicina continua a formatar moralmente as decisões e problemas em saúde, com implicaçóes para pacientes, médicos e instituiçôes de saúde.

Como guia analítico prático e de fácil compreensão para os profissionais da saúde, Gillon ${ }^{3}$ discute os quatro princípios e o escopo da ética médica: autonomia, beneficência, não-maleficência e justiça. Esta última se divide em justiça distributiva, justiça com base no direito e justiça legal. Esses quatro princípios fornecem um patamar de diálogo para diferentes culturas, crenças religiosas e posicionamentos políticos, visto que estes princípios são considerados prima facie: constituem dever que se impóe em todas as ocasióes em que se atua sobre a saúde das pessoas, a menos que haja um conflito entre deveres iguais ou mais fortes que estes. ${ }^{4}$ Assim, com base nesses quatro princípios que fundamentam a ética em medicina e, consequentemente, a aplicação da prevenção quaternária, pretende-se analisar criticamente o rastreamento do câncer de mama, enquanto medida preventiva.

Os programas organizados de rastreamento têm por princípio a utilização de um instrumento inicial de seleção ou peneiramento (i.e. mamografia) para separar pessoas assintomáticas na população-alvo, que necessitarão ser classificadas ou diagnosticadas por meio de um 'gold-standard' que define a doença (i.e. anatomopatológico) para, então, ser oferecido à pessoa o tratamento preventivo definitivo para a condição rastreada. ${ }^{5}$ Como esse tipo de intervenção recai sobre indivíduos saudáveis, os requerimentos éticos nos casos dos programas de rastreamento são altíssimos, pois os riscos de danos não estão contrabalançados com um sofrimento real (doença já instalada), mas sim, estão ancorados em um potencial futuro de adoecimento e morte. Neste caso, o princípio da não-maleficência (não causar danos) impera sobre o da beneficência (desejo de promover o bem-estar dos pacientes), visto que pessoas assintomáticas, que se percebem como saudáveis, podem ter sua saúde abalada indefinidamente devido a intervençáo da biomedicina. Os exemplos de danos mais citados na literatura são sofrimentos psicológicos (devido as incertezas dos falsos positivos, falsa segurança dos falsos negativos e das situaçōes limítrofes, que requerem monitoramento de perto, como as Neoplasias Intra-epiteliais Cervicais - NIC I, II, III), bem como as sequelas físicas resultantes dos tratamentos, tais como impotência ou incontinência urinária, no caso do rastreamento e tratamento do câncer de próstata. 
Como no rastreamento e/ou check up a intervenção é orientada com base em uma 'miragem' ou probabilidade, este pode resultar em 'danos sem potenciais benefícios', ${ }^{6}$ em que os procedimentos invasivos (para esclarecer 'imagens' ou resultados de exames 'positivamente' suspeitos na fase de seleção ou peneiramento) resultam em complicaçôes, porém a biópsia resulta normal. Por exemplo, as colonoscopias, laparoscopias, biópsias (de fígado, rim, próstata), podem produzir complicaçóes (perfuração de alça intestinal, complicaçáo na anestesia, perfuração de artéria importante, sepses) podendo escalonar para readmissão hospitalar, com estresse para pacientes e familiares e/ou em um pior cenário, morte do paciente com um laudo de anatomopatológico benigno. Portanto, os programas de rastreamento, por converterem pessoas saudáveis em enfermos em uma escala populacional, são altamente iatrogênicos, podendo ser resumidos na seguinte frase: "muito serão chamados, poucos os escolhidos...", mas muitos seräo prejudicados para que pouquissimos sejam 'curados'.

Isso é particularmente verdadeiro no caso do rastreamento do câncer de mama, que produz canceres fisiopatologicamente insignificantes (sobrediagnóstico) expondo mulheres previamente saudáveis a danos significativos devido ao tratamento com radioterapia. Gøtzsche et al. ${ }^{7}$ alertam para os riscos de efeitos adversos importantes da irradiação, tais como insuficiência cardíaca $(27 \%)$ por dano da circulação cardíaca e/ou indução de câncer de pulmão (78\%). Além do mais, uma revisão sistemática recentemente publicada no British Medical Journal ${ }^{\beta}$ sobre os efeitos adversos dos rastreamentos de cânceres em geral, verificou que somente um terço dos ensaios clínicos controlados aleatorizados se preocupou em medir os danos da intervenção do rastreamento. Esse artigo é importante porque afeta diretamente a prática dos profissionais para estabelecer os parâmetros de segurança da intervenção junto a seus pacientes, visto que existe um viés de seleçáo de informação que ressalta apenas os aspectos positivos do rastreamento, deixando de controlar e/ou monitorar potenciais danos.

Do ponto de vista ético, esse contexto de incerteza fere a autonomia das pacientes, criando muita vezes um falso empoderamento, uma vez que as mulheres não detém uma visão mais completa sobre os potenciais riscos e benefícios dos programas de rastreamento do câncer de mama. ${ }^{9}$ Para realmente empoderar a mulher, de modo a fortalecer sua autonomia para decidir sobre as intervençóes que afetam sua saúde, há a necessidade de que a informação seja mais transparente e que revele também os potenciais danos da intervenção. Além disso, a linguagem usada na divulgação da informação deve ser neutra, de simples entendimento, culturalmente acessível, de modo que as usuárias do sistema de saúde possam decidir melhor sobre sua saúde. ${ }^{3}$

Do ponto de vista da saúde pública, da ética da justiça distributiva, e dos limitados recursos em saúde que qualquer sistema de saúde enfrenta, os programas de rastreamento desviam recursos financeiros - que deveriam ser prioritariamente investidos no tratamento e cuidado das pessoas doentes - para as pessoas saudáveis, com o agravo de produzir novos doentes reais, fruto do dano da intervenção sobre corpos saudáveis, gerando mais custos para o sistema de saúde e para a sociedade em geral.

Felizmente, os programas de rastreamento estáo cada vez mais perdendo sua força, principalmente na Europa, a exemplo do Swiss Medical Board ${ }^{10}$ que não encontrou razão para a manutenção dos programas de rastreamento do câncer de mama, em face das novas evidências científicas. Na Dinamarca a taxa de mortalidade atribuída ao câncer de mama não foi reduzida devido a implementação do rastreamento sistemático do câncer de mama com mamografias, ao longo de 17 anos de seguimento, ${ }^{11}$ entretanto, se produziu uma taxa de sobrediagnóstico de $33 \% .{ }^{12}$ Resultados semelhantes também foram encontrados nos Estados Unidos após 30 anos de observação ${ }^{13}$, e no Canadá, o acumulado de 25 anos de acompanhamento dos efeitos do rastreamento do câncer de mama, além de não representar redução da mortalidade por câncer de mama, resultou em $22 \%$ de sobrediagnósticos. ${ }^{14}$ Assim, para Peter C. Gøtzsche, ${ }^{1}$ umas das maiores autoridades mundiais sobre o tema, o melhor método que dispomos para reduzir a ocorrência do câncer de mama é parar com o seu rastreamento por meio de mamografias.

Desse modo, tanto do ponto de vista ético como científico, ${ }^{10}$ os programas de rastreamento deveriam ser descontinuados ou se restringirem a grupos ou situações muito específicas, e o foco da prevenção ser redirecionado para a intervenção no sintomático-precoce, visto que o tratamento do câncer de mama melhorou consideravelmente nas últimas décadas, sendo este o provável responsável pela melhoria da qualidade de vida das mulheres afetadas. ${ }^{1}$ A Força Tarefa Canadense ${ }^{15}$ de cuidados preventivos em saúde, em sua mais recente atualização (em 2011) avaliou como fraca a recomendação para o rastreamento do câncer de mama com mamografia a cada 2 a 3 anos na faixa etária de 50 a 69 anos, pois as evidências para o rastreio foram consideradas apenas de moderada qualidade. Assim, o Ministério da Saúde ${ }^{16}$ brasileiro agiu de forma bem fundamentada ao restringir os incentivos financeiros ao rastreamento do câncer de mama à faixa etária de 50 a 69 anos.

Portanto, 'não há nada de errado em dizer não à mamografia', ${ }^{9}$ pois, ao se atuar sobre pessoas assintomáticas e saudáveis, o princípio da não-maleficência deve sobrepor-se ao da beneficência. $O$ desafio posto aos médicos de família e comunidade é o de individualizar cada caso neste mar de incertezas, compartilhando com seus pacientes os potenciais danos, frequentemente omitidos, atribuídos ao rastreamento de cânceres, de modo a operacionalizar na prática a prevenção quaternária. 


\section{Referências}

1. Gøtzsche PC. Time to stop mammography screening? CMAJ. 2011;183(17):1957-8. http://dx.doi.org/10.1503/cmaj.111721

2. Norman $A H$, Tesser CD. Prevenção quaternária na atenção primária à saúde: uma necessidade do Sistema Único de Saúde. Cad Saúde Pública. 2009;25(9):2012-20. http://dx.doi.org/10.1590/S0102-311X2009000900015

3. Gillon R. Medical ethics: four principles plus attention to scope. BMJ. 1994;309(6948):184. http://dx.doi.org/10.1136/bmj.309.6948.184

4. Limentani AE. The role of ethical principles in health care and the implications for ethical codes. J Med Ethics. 1999;25:394-8. http://dx.doi. org/10.1136/jme.25.5.394

5. Norman AH, Tesser CD. Rastreamento de doenças. In Lopes JMC, Gusso GDF, editors. Tratado de Medicina de Família e Comunidade: princípios, formação e prática. Porto Alegre: Artmed; 2012. Vol 1. p. 521-32

6. Gray JAM. New concepts in screening. B J Gen Pract. 2004;54(501):292-8. PMCID: PMC1326079

7. Gøtzsche PC, Jørgensen KJ. Screening for breast cancer with mammography. The Cochrane Database of Systematic Reviews. (2013);6:CD001877. http://dx.doi.org/10.1002/14651858.CD001877.pub5

8. Heleno B, Thomsen MF, Rodrigues DS, Jorgensen KJ, Brodersen J. Quantification of harms in cancer screening trials: literature review. BMJ. 2013;347:5334. http://dx.doi.org/10.1136/bmj.f5334

9. Heath I. It is not wrong to say no. BMJ. 2009;338:2529. http://dx.doi.org/10.1136/bmj.b2529

10. Biller-Andorno N, Jüni P. Abolishing mammography screening programs? A view from the swiss medical board. N England J Med. $2014 ; 1-3$. http://dx.doi.org/10.1056/NEJMp1401875

11. Jørgensen KJ, Zahl PH, Gøtzsche PC. Breast cancer mortality in organised mammography screening in Denmark: comparative study. BMJ. 2010;340,c1241. http://dx.doi.org/10.1136/bmj.c1241

12. Jørgensen KJ, Zahl PH, Gøtzsche PC. Overdiagnosis in organised mammography screening in Denmark: a comparative study. BMC Women's Health. 2009;9:36. http://dx.doi.org/10.1186/1472-6874-9-36

13. Bleyer A, Welch HG. Effect of three decades of screening mammography on breast-cancer incidence. N England J Med. 2012;367(21):19982005. http://dx.doi.org/10.1056/NEJMoa1206809

14. Miller AB, Wall C, Baines CJ, Sun P, To T, Narod SA. Twenty-five year follow-up for breast cancer incidence and mortality of the Canadian National Breast Screening Study: randomised screening trial. BMJ. 2014;348:g366. http://dx.doi.org/10.1136/bmj.g366

15. Ministério da Saúde (BR), Secretaria de Atenção Básica. Nota técnica conjunta: Rastreamento do câncer de mama. Brasília: MS; 2013 [Acesso em 2014 Apr 14]. Disponível em: http://sbmfc.org.br/media/Nota_CGAPDC_CPV.pdf

16. Canadian Task Force on Preventive Health Care. Screening for Breast Cancer: summary of recommendations for clinicians and policy-makers. Canadá; c2000-2014 [acesso em 2014 Apr 14]. Disponível em: http://canadiantaskforce.ca/guidelines/2011-breast-cancer/. 